\title{
Refined Prediction of Pharmacokinetic Kratom-Drug Interactions: Time-Dependent Inhibition Considerations $\mathbf{s}$
}

\author{
Rakshit S. Tanna, Dan-Dan Tian, ${ }^{1}$ Nadja B. Cech, Nicholas H. Oberlies, Allan E. Rettie, \\ Kenneth E. Thummel, and Mary F. Paine \\ Department of Pharmaceutical Sciences, College of Pharmacy and Pharmaceutical Sciences, Washington State University, \\ Spokane, Washington (R.S.T., D.-D.T., M.F.P.); Department of Chemistry and Biochemistry, University of North Carolina at \\ Greensboro, Greensboro, North Carolina (N.B.C., N.H.O.); Departments of Medicinal Chemistry (A.E.R.) and Pharmaceutics \\ (K.E.T.), School of Pharmacy, University of Washington, Seattle, Washington; and Center of Excellence for Natural Product Drug \\ Interaction Research, Spokane, Washington (N.B.C., N.H.O., A.E.R., K.E.T., M.F.P.)
}

Received August 5, 2020; accepted October 8, 2020

\begin{abstract}
Preparations from the leaves of the kratom plant (Mitragyna speciosa) are consumed for their opioid-like effects. Several deaths have been associated with kratom used concomitantly with some drugs. Pharmacokinetic interactions are potential underlying mechanisms of these fatalities. Accumulating in vitro evidence has demonstrated select kratom alkaloids, including the abundant indole alkaloid mitragynine, as reversible inhibitors of several cytochromes P450 (CYPs). The objective of this work was to refine the mechanistic understanding of potential kratomdrug interactions by considering both reversible and timedependent inhibition (TDI) of CYPs in the liver and intestine. Mitragynine was tested against CYP2C9 (diclofenac 4'-hydroxylation), CYP2D6 (dextromethorphan O-demethylation), and CYP3A (midazolam 1'-hydroxylation) activities in human liver microsomes (HLMs) and CYP3A activity in human intestinal microsomes (HIMs). Comparing the absence to presence of NADPH during preincubation of mitragynine with HLMs or HIMs, an $\sim 7$-fold leftward shift in $\mathrm{IC}_{50}(\sim 20$ to $3 \mu \mathrm{M})$ toward CYP3A resulted, prompting determination of TDI parameters (HLMs: $K_{l}$, $4.1 \pm 0.9 \mu \mathrm{M} ; K_{\text {inact }}, 0.068 \pm 0.01 \mathrm{~min}^{-1} ; \mathrm{HIMs}: K_{l}, 4.2 \pm 2.5 \mu \mathrm{M}$; $\left.k_{\text {inact }}, 0.079 \pm 0.02 \mathrm{~min}^{-1}\right)$. Mitragynine caused no leftward shift
\end{abstract}

in IC I0 $_{50}$ toward CYP2C9 $(\sim 40 \mu \mathrm{M})$ and CYP2D6 $(\sim 1 \mu \mathrm{M})$ but was a strong competitive inhibitor of CYP2D6 $\left(K_{i}, 1.17 \pm 0.07 \mu \mathrm{M}\right)$. Using a recommended mechanistic static model, mitragynine (2$\mathrm{g}$ kratom dose) was predicted to increase dextromethorphan and midazolam area under the plasma concentration-time curve by 1.06- and 5.69-fold, respectively. The predicted midazolam area under the plasma concentration-time curve ratio exceeded the recommended cutoff (1.25), which would have been missed if TDI was not considered.

\section{SIGNIFICANCE STATEMENT}

Kratom, a botanical natural product increasingly consumed for its opioid-like effects, may precipitate potentially serious pharmacokinetic interactions with drugs. The abundant kratom indole alkaloid mitragynine was shown to be a time-dependent inhibitor of hepatic and intestinal cytochrome P450 3A activity. A mechanistic static model predicted mitragynine to increase systemic exposure to the probe drug substrate midazolam by 5.7-fold, necessitating further evaluation via dynamic models and clinical assessment to advance the understanding of consumer safety associated with kratom use.

\section{Introduction}

Kratom [Mitragyna speciosa (Korth.) Havil.] is a tropical tree belonging to the coffee family (Rubiaceae) that is indigenous to Southeast Asia (https://www.drugabuse.gov/ publications/drugfacts/kratom). Kratom leaves are reported to elicit both stimulant and relaxant/analgesic properties

This work was supported by the National Institutes of Health National Center for Complimentary and Integrative Health [Grant U54-AT008909] (to M.F.P.). The content is solely the responsibility of the authors and does not necessarily represent the official views of NIH.

${ }^{1}$ Current affiliation: Drug Disposition, Eli Lilly and Company, Indianapolis, Indiana.

https://doi.org/10.1124/jpet.120.000270.

S This article has supplemental material available at jpet.aspetjournals.org.
(Chang-Chien et al., 2017). Historically, agricultural laborers in Thailand and Malaysia chewed the leaves to increase stamina (Cinosi et al., 2015). Consuming kratom leaves in higher quantities has also been shown to elicit opioid-like effects. Based on these latter effects, Malaysian authorities deemed kratom to have addiction potential, prohibiting kratom use in that country (Khalil et al., 2020). The legal status of kratom varies in other countries depending on region and, in the United States, by state, city, and county (Veltri and Grundmann, 2019). Accordingly, sales in the United States and several European countries continue to flourish (Henningfield et al., 2018). Despite increasing sales, the pharmacological effects, safety, and addiction potential of kratom remain understudied.

ABBREVIATIONS: $\mathrm{AUC}_{\mathrm{R}}$, ratio of area under the plasma concentration-time curve in presence to absence of inhibitor; CYP, cytorchrome P450; $F_{g}$, fraction of the object drug escaping metabolism in the gut; $f_{m}$, fraction of the object drug metabolized via a particular pathway; $f_{u, p}$, fraction unbound in human plasma; HIM, human intestinal microsome; HLM, human liver microsome m/z mass to charge ratio; TDI, time-dependent inhibition; UPLC-MS/MS, ultra-high-performance liquid chromatography-tandem mass spectrometry. 
TABLE 1

Major enzymes involved in the metabolism of opioids and/or other drugs detected in postmortem blood samples obtained from kratom-related death cases in Colorado (Gershman et al., 2019)

\begin{tabular}{|c|c|c|c|c|}
\hline $\begin{array}{l}\text { Case } \\
\text { no. }\end{array}$ & $\begin{array}{l}\text { Blood Mitragynine } \\
\text { Concentration }(\mu \mathrm{M})\end{array}$ & Other Drugs & Major Enzyme(s) & References \\
\hline 1 & Positive $^{\mathrm{a}}$ & $\begin{array}{c}\text { Butyryl-fentanyl, oxycodone, etizolam, } \\
\text { diphenhydramine, THC }\end{array}$ & CYP3A, CYP2D6 & $\begin{array}{l}\text { Lalovic et al. (2004); Niwa et al. (2005); } \\
\text { Kanamori et al. (2019) }\end{array}$ \\
\hline 2 & Positive & Oxycodone, fluoxetine, pseudoephedrine & CYP3A, CYP2D6 & $\begin{array}{l}\text { Lalovic et al. (2004); LLerena et al. } \\
\text { (2004) }\end{array}$ \\
\hline 3 & 0.04 & Etizolam, 5-MeO-AMT & CYP3A & Niwa et al. (2005) \\
\hline 4 & Positive & Morphine, codeine & UGT2B7, CYP3A, CYP2D6 & Crews et al. (2014) \\
\hline 5 & 0.35 & $\begin{array}{l}\text { Oxycodone, tramadol, topiramate, } \\
\text { diphenhydramine, zolpidem }\end{array}$ & CYP3A, CYP2D6 & $\begin{array}{l}\text { Pichard et al. (1995); Miotto et al. } \\
\text { (2017); Yamamoto et al. (2017) }\end{array}$ \\
\hline 6 & 5.3 & Citalopram & CYP3A, CYP2C19, CYP2D6 & von Moltke et al. (2001) \\
\hline 7 & 3.5 & Furanyl-fentanyl & NA & NA \\
\hline 8 & 2.5 & $\begin{array}{c}\text { Temazepam, olanzapine, sertraline, } \\
\text { clonazepam }\end{array}$ & $\begin{array}{c}\text { CYP3A, CYP2C9, } \\
\text { CYP2C19, CYP2D6, } \\
\text { UGT2B7 }\end{array}$ & $\begin{array}{c}\text { Kobayashi et al. (1999); Urichuk et al. } \\
\text { (2008); Tóth et al. (2016) }\end{array}$ \\
\hline 9 & 0.42 & 3,4-Dimethoxy- $N$-methylamphetamine & NA & NA \\
\hline 10 & 6.7 & Etizolam, nordiazepam, mirtazapine & CYP3A, CYP2D6, CYP1A2 & $\begin{array}{c}\text { Ono et al. (1996); Störmer et al. (2000); } \\
\text { Niwa et al. (2005) }\end{array}$ \\
\hline 11 & 12 & 2,4,5-Trimethoxyamphetamine & CYP2D6 & Ewald and Maurer (2008) \\
\hline 12 & 0.62 & Oxycodone & CYP3A, CYP2D6 & Lalovic et al. (2004) \\
\hline 13 & 1.9 & Oxycodone, fentanyl, cocaine & CYP3A, CYP2D6, esterases & $\begin{array}{l}\text { Kamendulis et al. (1996); Guitton et al. } \\
\text { (1997); Kanamori et al. (2019) }\end{array}$ \\
\hline 14 & Positive & $-^{\mathrm{b}}$ & - & - \\
\hline 15 & Positive & $\mathrm{U}-47700$ & NA & NA \\
\hline
\end{tabular}

5-MeO-AMT, 5-methoxy- $\alpha$-methyltryptamine; NA, information not available; THC, tetrahydrocannabinol; U-47700, synthetic opioid; UGT, uridine diphosphateglucuronyltransferase.

${ }^{a}$ Qualitative detection.

${ }^{b}$ No other drugs reported.

The opioid-like effects of kratom are attributed to indole alkaloids contained in the leaves (Kruegel et al., 2016). Mitragynine, typically the most abundant (up to $66 \%$ of total alkaloid content), and 7-hydroxymitragynine are the most extensively studied (Hassan et al., 2013). Both alkaloids have been shown to bind to multiple opioid receptors, of which the potencies and functional activities vary with in vitro system and in vivo animal model (Kruegel and Grundmann, 2018). Based on these biologic effects, kratom leaves are promoted for self-management of opioid withdrawal symptoms and pain (Throckmorton et al., 2018; Williams and Nikitin, 2020). In 2016, the US Drug Enforcement Administration's intent to classify mitragynine and 7-hydroxymitragynine as schedule I controlled substances was later withdrawn because of public outcry about these purported medicinal benefits (https://www. dea.gov/press-releases/2016/08/30/dea-announces-intentschedule-kratom).

Although kratom may have medicinal value, calls to poison centers in the United States involving kratom exposures increased annually from 2011 to 2017 (13 to 682), one-third of which reported use with drugs of abuse, including several opioids and benzodiazepines (Post et al., 2019). Blood autopsy of 14 out of 15 overdose deaths in Colorado associated with kratom use tested positive for these and other drugs (Table 1) (Gershman et al., 2019). According to a Centers for Disease Control and Prevention report (Olsen et al., 2019), 152 overdose deaths from July 2016 to December 2017 tested positive for mitragynine. However, only seven decedents tested negative for the presence of concomitant drugs upon postmortem toxicology reports. Although these deaths could have been caused by kratom or the known (or unknown) concomitant $\operatorname{drug}(\mathrm{s})$, the collective evidence suggests that these deaths might have involved kratom-drug interactions, which may be pharmacodynamic and/or pharmacokinetic in nature.
As with drug-drug interactions, common mechanisms underlying natural product-drug interactions include inhibition of drug metabolizing enzymes, particularly the CYPs, by precipitant ("perpetrator") constituents in natural products (Johnson et al., 2018; Paine et al., 2018). Kratom extracts, when tested using recombinant enzymes with fluorometric or luminogenic CYP probe substrates, inhibited CYP2D6, CYP3A, and CYP1A2 activity $\left(\mathrm{IC}_{50}\right.$ : 0.64-3.6, 0.78-140, and $39 \mu \mathrm{g} / \mathrm{ml}$, respectively) (Kong et al., 2011; Hanapi et al., 2013). Mitragynine similarly showed the strongest inhibition toward CYP2D6 activity $\left(\mathrm{IC}_{50}, 2.2 \mu \mathrm{M}\right)$ in human liver microsomes (HLMs) relative to other CYPs, including CYP2C19, CYP3A, and CYP2C8 $\left(\mathrm{IC}_{50}, 10.5-33.5 \mu \mathrm{M}\right)$. Modest to no inhibitory effects were observed with other tested CYPs, including CYP1A2 and CYP2C9 ( $\mathrm{IC}_{50}>45 \mu \mathrm{M}$ ) (Kamble et al., 2020).

Using an in vitro to in vivo extrapolation approach, mitragynine was predicted to precipitate clinical pharmacokinetic interactions with drugs cleared only by CYP2D6 (area under the plasma concentration-time curve ratio, 1.2-12) (Kamble et al., 2020). However, this observation does not explain the toxic concentrations of the antipsychotic and CYP3A substrate quetiapine detected in the postmortem blood evaluation of a young man who tested positive for mitragynine and who was believed not to have ingested excessive quantities of quetiapine based on pill count (Hughes, 2019). Because quetiapine undergoes extensive first-pass metabolism in at least the liver (Gjestad et al., 2017), these toxic concentrations could have involved time-dependent inhibition (TDI) of CYP3A. Such inhibition produces an altered enzyme state that persists longer than that with reversible inhibition, leading to a clinically significant drug interaction.

The objective of this work was to reassess the pharmacokinetic drug interaction potential of kratom using mitragynine 


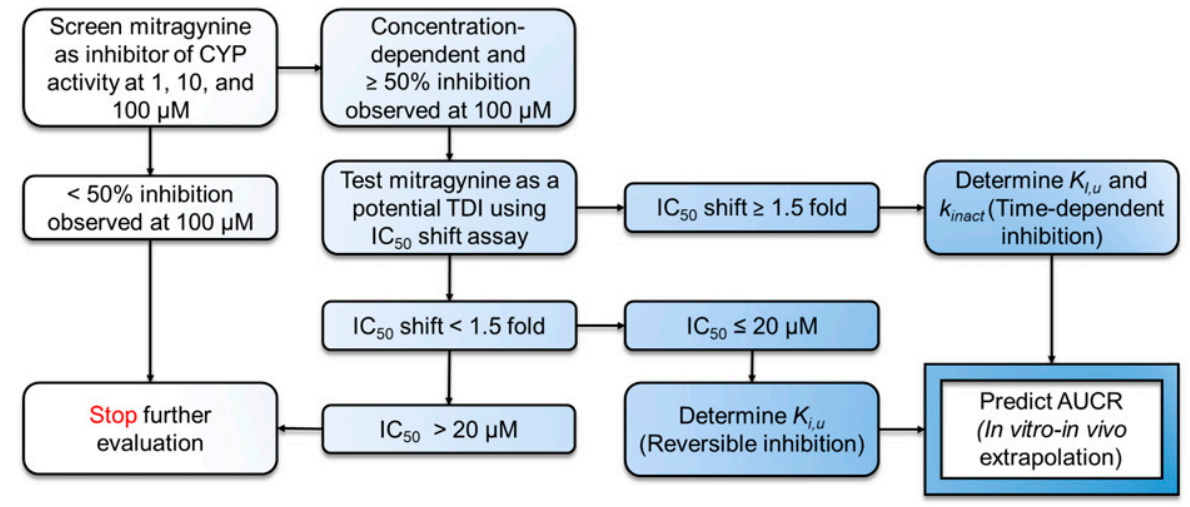

Fig. 1. Workflow for reevaluating CYP-mediated drug interaction risk associated with kratom use using mitragynine as the marker constituent. Inhibition of a CYP in a concentration-dependent manner and by at least $50 \%$ at the highest tested concentration indicates mitragynine-mediated inhibition. A leftward shift of $\geq 1.5$-fold in $\mathrm{IC}_{50}$ indicates potential TDI (Grimm et al., 2009). An $\mathrm{IC}_{50}$ value $<20 \mu \mathrm{M}$ indicates potential clinical relevance of CYP inhibition, determined relative to the highest mitragynine concentration quantified from autopsy blood samples.

as a marker constituent and an in vitro to in vivo extrapolation approach that included both reversible and TDI of select CYPs in the liver and intestine (Fig. 1). The aims were to 1) screen kratom extracts and mitragynine as inhibitors of major CYPs collectively responsible for the metabolism of $\sim 70 \%$ of the top 200 most prescribed drugs (CYP2C9, CYP2D6, and CYP3A) (Saravanakumar et al., 2019); 2) evaluate mitragynine as a potential time-dependent inhibitor of select enzymes based on predefined cutoffs; 3 ) determine the inhibition kinetics (reversible, time-dependent) for mitragynine toward select CYPs based on predefined cutoffs; and 4) predict the magnitude of change in the systemic exposure to select object drugs using mechanistic static models. Results provide critical mechanistic data to inform the need for further in vivo evaluation of risks associated with kratom consumption with drugs metabolized by CYP2C9, CYP2D6, and CYP3A.

\section{Materials and Methods}

\section{Materials and Chemicals.}

Dextromethorphan, dextrorphan, diclofenac, and 4'-hydroxydiclofenac were purchased from Toronto Research Chemicals Inc. (Toronto, Canada). Midazolam, 1'-hydroxymidazolam, sulfaphenazole, and NADPH were purchased from Cayman Chemical (Ann Arbor, MI). Alprazolam, ketoconazole, and quinidine were purchased from SigmaAldrich (St. Louis, MO). HLMs (H0604, mixed gender, pool of 15, lot 1010191) and HIMs (H0610.I, mixed gender, pool of 10, lot 1610314) were purchased from XenoTech, LLC (Kansas City, KS). Potassium phosphate buffer salts were purchased from Fisher Scientific (Fair Lawn, NJ). All other chemicals and reagents were analytical-grade.

\section{Screening of Kratom Extracts and Mitragynine as Inhibitors of CYP2C9, CYP2D6, and CYP3A Activity.}

Methanolic extracts were prepared from three commercially available kratom products (coded K-50, K-51, and K-52) as described (Flores-Bocanegra et al., 2020; (Todd et al., 2020). The extracts and mitragynine were tested as inhibitors of CYP2C9, CYP2D6, and CYP3A activities using a cocktail approach (McDonald et al., 2015, $2020)$ with modifications. In brief, a kratom extract $(2,10,20 \mu \mathrm{g} / \mathrm{ml})$ or mitragynine $(1,10,100 \mu \mathrm{M})$ was incubated at $37^{\circ} \mathrm{C}$ in 96 -well plates with HLMs or HIMs $(0.05 \mathrm{mg} / \mathrm{ml})$ and the probe substrates diclofenac (CYP2C9, $4 \mu \mathrm{M}$ ), dextromethorphan (CYP2D6, $4 \mu \mathrm{M}$ ), and midazolam (CYP3A, $2 \mu \mathrm{M})$ in potassium phosphate buffer (0.1 M, pH 7.4); the final organic (methanol) concentration $(\mathrm{v} / \mathrm{v})$ and incubation volume was $<0.8 \%$ and $400 \mu \mathrm{l}$, respectively. After 5 minutes of equilibration, reactions were initiated by adding NADPH (final concentration, 1 $\mathrm{mM}$ ). After 2 minutes (CYP3A activity in HLMs) or 10 minutes
(CYP2C9 and CYP2D6 activity in HLMs, CYP3A activity in HIMs), reactions were terminated with two volumes of ice-cold methanol containing internal standard (alprazolam, $100 \mathrm{nM}$ ). Metabolite formation was linear with respect to time for all experiments (data not shown). The quenched mixtures were centrifuged at $2270 \mathrm{~g}$ for 10 minutes, and the supernatants were subjected to UPLC-MS/MS analysis for quantification of $4^{\prime}$-hydroxydiclofenac, dextrorphan, and $1^{\prime}$-hydroxymidazolam (described below).

\section{$I_{50}$ Shift Determination for CYP2C9, CYP2D6, and CYP3A Activities.}

Mitragynine (final concentration, $0.015-100 \mu \mathrm{M}$ ) was incubated at $37^{\circ} \mathrm{C}$ in 96 -well plates with HLMs or HIMs $(0.05 \mathrm{mg} / \mathrm{ml})$ in the presence or absence of $1 \mathrm{mM}$ NADPH in potassium phosphate buffer; the final methanol concentration and incubation volume was $<0.8 \%$ and $250 \mu \mathrm{l}$, respectively. After 30 minutes, $196 \mu \mathrm{l}$ of the primary incubation mixture was transferred to a clean 96 -well plate containing $2 \mu \mathrm{l}$ of concentrated probe substrates (final concentration: diclofenac, $4 \mu \mathrm{M}$; dextromethorphan, $4 \mu \mathrm{M}$; midazolam, $2 \mu \mathrm{M})$ plus $2 \mu \mathrm{l}$ of potassium phosphate buffer $(100 \mathrm{mM})$ or NADPH (final concentration, $1 \mathrm{mM}$ ). After 2 minutes (CYP3A activity in HLMs) or 10 minutes (CYP2C9 and CYP2D6 activity in HLMs, CYP3A activity in HIMs), the secondary reaction was terminated and processed for analysis by UPLC-MS/MS in the same manner as described for the initial screen. IC $_{50}$ values were recovered as described (Gufford et al., 2014; Tian et al., 2018) via nonlinear least-squares regression using Phoenix (v8.2; Certara, Princeton, NJ).

\section{$K_{i}$ Determination toward CYP2D6 Activity.}

Mitragynine (final concentration, $0.12-10 \mu \mathrm{M}$ ) was incubated at $37^{\circ}$ $\mathrm{C}$ in 96-well plates with HLMs $(0.05 \mathrm{mg} / \mathrm{ml})$, dextromethorphan (final concentration, 1.85-50 $\mu \mathrm{M}$ ), and potassium phosphate buffer; the final methanol concentration and incubation volume was $<0.5 \%$ and $250 \mu \mathrm{l}$, respectively. After 5 minutes of equilibration, reactions were initiated by adding NADPH (final concentration, 1 $\mathrm{mM}$ ). After 10 minutes, reactions were terminated and processed for analysis by UPLC-MS/MS for dextrorphan in the same manner as described for the initial screen. $K_{i}$ values were determined by fitting appropriate models (competitive inhibition, uncompetitive inhibition, mixed inhibition) as described (Gufford et al., 2015; Tian et al., 2018) via nonlinear least-squares regression using Phoenix.

\section{$K_{l}$ and $\boldsymbol{k}_{\text {inact }}$ Determination toward CYP3A Activity.}

Nondilution Approach. Mitragynine (final concentration, 0.56-30 $\mu \mathrm{M})$ was incubated at $37^{\circ} \mathrm{C}$ in 96 -well plates with HLMs or HIMs $(0.05 \mathrm{mg} / \mathrm{ml})$ in the presence of $1 \mathrm{mM} \mathrm{NADPH}$ in potassium phosphate buffer; the final methanol concentration and incubation volume was $<0.5 \%$ and $1100 \mu \mathrm{l}$, respectively. After $0,2,5,10$, and 20 minutes, $196 \mu \mathrm{l}$ 
of the primary incubation mixture was transferred to a clean 96 -well plate containing $2 \mu \mathrm{l}$ of the concentrated probe substrate (final concentration, $20 \mu \mathrm{M}$ ) plus $2 \mu \mathrm{l}$ of potassium phosphate buffer or NADPH (final concentration, $1 \mathrm{mM}$ ). After 5 minutes, the secondary incubation was terminated and processed for analysis by UPLC-MS/ MS for 1'-hydroxymidazolam in the same manner as described for the initial screen.

Dilution Approach. Mitragynine (final concentration, 3-60 $\mu \mathrm{M}$ ) was incubated at $37^{\circ} \mathrm{C}$ in 96 -well plates with HLMs $(0.5 \mathrm{mg} / \mathrm{ml})$ in the presence of $1 \mathrm{mM}$ NADPH in potassium phosphate buffer; the final methanol concentration and incubation volume was $<0.5 \%$ and $200 \mu \mathrm{l}$, respectively. After 0,5 , and 20 minutes, $20 \mu \mathrm{l}$ of the primary incubation mixture were transferred to a clean 96 -well plate containing $380 \mu \mathrm{l}$ of midazolam (final concentration, $10 \mu \mathrm{M}$ ) and NADPH (final concentration, $1 \mathrm{mM}$ ) in potassium phosphate buffer. After 5 minutes, the secondary incubation was terminated and processed as described for the nondilution approach. $K_{I}$ and $k_{\text {inact }}$ were recovered as described (Ainslie et al., 2014) via nonlinear least-squares regression using Phoenix.

\section{UPLC-MS/MS Analysis for $4^{\prime}$-Hydroxydiclofenac, Dextrorphan, and 1'-Hydroxymidazolam.}

A Shimadzu Nexera X2 UPLC (Shimadzu Corporation, Tokyo, Japan) interfaced with the QTRAP system (AB Sciex, Framingham, MA) operating in positive electrospray ionization mode was used to quantify all metabolites. Chromatographic separation was achieved using a reverse-phase column (Acquity $\mathrm{C}_{18}$ column, $3 \mu \mathrm{M}$, $50 \times 2.1 \mathrm{~mm}$; Thermo Scientific, Waltham, MA) with a guard column, heated to $40^{\circ} \mathrm{C}$, and a binary gradient consisting of $0.1 \%$ formic acid in water (A) and $0.1 \%$ formic acid in methanol (B). The following gradient was applied: $0-0.4$ minutes, $10 \% \mathrm{~B}$; 0.4-1.0 minutes, $10 \%-95 \% \mathrm{~B}$; $1.0-2.0$ minutes, $95 \% \mathrm{~B} ; 2.0-2.1$ minutes, $95 \%-10 \% \mathrm{~B}$; and $2.1-3.0$ minutes, $10 \% \mathrm{~B}$. The following mass transitions were monitored: $\mathrm{m} / \mathrm{z} 312.0 \rightarrow 231.0$ ( $4^{\prime}$ 'hydroxydiclofenac), $\mathrm{m} / \mathrm{z} 258.2 \rightarrow 157.2$ (dextrorphan), $\mathrm{m} / \mathrm{z} 342.0 \rightarrow 324.0$ ( $1^{\prime}$-hydroxymidazolam), and $\mathrm{m} / \mathrm{z} 309.1 \rightarrow 281.0$ (alprazolam). All metabolite concentrations were quantified using MultiQuant software (version 2.1.1; AB Sciex) by interpolation from matrixmatched calibration curves (1.37-1000 nM) prepared using standards. The accuracy of all the calibration standards and quality controls were within $100 \% \pm 20 \%$ at the low limit of quantification or $100 \% \pm 15 \%$ at levels above the low limit of quantification.

\section{In Vitro to In Vivo Predictions.}

The in vivo kratom-drug interaction risk was predicted using mitragynine as a marker constituent and the following mechanistic static model (Food and Drug Administration Center for Drug Evaluation and Research, 2020):

$A U C R=\left(\frac{1}{\left[A_{g} \times B_{g}\right] \times\left(1-F_{g}\right)+F_{g}}\right) \times\left(\frac{1}{\left[A_{h} \times B_{h}\right] \times f_{m} \times\left(1-f_{m}\right)}\right)$,

where AUCR denotes the ratio of the area under the plasma versus concentration time curve for the object drug in the presence to absence of inhibitor (mitragynine). $A_{g}$ (eq. 2 ) and $B_{g}$ (eq. 3) represent the effects of reversible inhibition and time-dependent inhibition in the gut, respectively; $A_{h}$ (eq. 4) and $B_{h}$ (eq. 5) represent the effects of reversible inhibition and time-dependent inhibition in the liver, respectively. $F_{g}$ is the fraction of the object drug escaping metabolism in the gut, and $f_{m}$ is the fraction of the object drug metabolized by the affected pathway. $F_{g}$ and $f_{m}$ for midazolam were set at 0.51 and 0.93 , respectively (Ramsden et al., 2019); $f_{m}$ for dextromethorphan was set at 0.95 (Parmentier et al., 2019).

$$
\begin{aligned}
& A_{g}=\frac{1}{1+\frac{\left[I I_{g}\right.}{K_{i, u}}} \\
& B_{g}=\frac{k_{\text {deg g }}}{k_{\text {deg g. }}+\frac{I I g_{g} \times k_{\text {inact }}}{\mid I]_{g}+K_{I, u}}} \\
& A_{h}=\frac{1}{1+\frac{\left[I I_{h}\right.}{K_{i, u}}} \\
& B_{h}=\frac{k_{\text {deg }, h}}{k_{\text {deg }, h}+\frac{[I]_{h} \times k_{\text {inact }}}{\left[I_{h}+K_{I, u}\right.}}
\end{aligned}
$$

$k_{\text {inact }}, K_{i, u}$, and $K_{I, u}$ represent the maximum rate of inactivation, unbound reversible inhibition constant, and TDI constant, respectively. $K_{i}$ and $K_{I}$ were corrected for microsomal binding (fraction of mitragynine unbound in microsomes, 0.96 ) generated in silico (GastroPlus; Simulations Plus Inc., Lancaster, CA). $k_{\text {deg }}$ is the degradation rate constant for the affected CYP in the liver and gut. $k_{\text {deg }}$ values for CYP3A in the liver and gut were set at 0.00032 and $0.00048 \mathrm{~min}^{-1}$, respectively (Fahmi et al., 2009). $[I]_{g}$ (eq. 6) and $[I]_{h}$ (eq. 7) represent the concentration of inhibitor in enterocytes and unbound inhibitor concentration in the portal vein, respectively.

$$
\begin{aligned}
{[I]_{g} } & =\frac{\text { Dose } \times k_{a} \times F_{a}}{Q_{e n}} \\
{[I]_{h} } & =f_{u, p} \times\left(C_{\text {max }}+\frac{\text { Dose } \times k_{a} \times F_{a}}{Q_{h} \times R_{b}}\right),
\end{aligned}
$$

where $f_{u, p}$ is the fraction unbound for inhibitor in human plasma; that for mitragynine was set at 0.02 (Obeng et al., 2020). Hepatic blood flow $\left(Q_{h}\right)$ and enterocyte blood flow $\left(Q_{e n}\right)$ were set at 97 and $181 / \mathrm{h}$ per $70 \mathrm{~kg}$, respectively. The first-order absorption rate $\left(k_{a}\right)$ was assumed to be $0.1 \mathrm{~min}^{-1}$. The human blood-to-plasma ratio $\left(R_{b}\right)$ and fraction absorbed into enterocytes $\left(F_{a}\right)$ were assumed to be 1 . Based on results with midazolam, kratom-drug interactions involving other CYP3A substrates were predicted to assess the AUCR associated with CYP3A TDI by mitragynine. Test object drugs were selected that encompassed a wide range of $F_{g}$ and $f_{m}$ values (Table 3 ).

\section{Results}

Kratom Extracts and Mitragynine Differentially Inhibited CYP2C9, CYP2D6, and CYP3A Activity. All three methanolic kratom extracts and mitragynine inhibited CYP2C9, CYP2D6, and CYP3A activities in HLMs in a concentration-dependent manner (Fig. 1; Supplemental Data). The extracts inhibited a given CYP by similar extents and were the most potent toward CYP2D6. The extracts at the lowest tested concentration $(2 \mu \mathrm{g} / \mathrm{ml})$ inhibited CYP2D6, CYP2C9, and CYP3A by 44\%-64\%, 24\%-29\%, and 15\%-23\%, respectively. Mitragynine at the lowest tested concentration $(1 \mu \mathrm{M})$ inhibited these enzymes by $57 \%, 21 \%$, and $26 \%$, respectively. The three kratom extracts and mitragynine inhibited CYP3A activity in HIMs in a concentrationdependent manner (Fig. 1; Supplemental Data), and the extent of inhibition was relatively higher in HIMs compared with HLMs at the higher tested concentrations. Kratom extracts and mitragynine at the lowest tested concentration inhibited CYP3A activity in HIMs by $24 \%-25 \%$ and $~ 9 \%$, respectively.

IC $_{50}$ Shift Experiments Suggested Mitragynine Is a Time-Dependent Inhibitor of CYP3A but Not of CYP2C9 or CYP2D6 Activity. Compared with the absence of NADPH during the 30-minute preincubation of mitragynine 
TABLE 2

$\mathrm{IC}_{50}$ for mitragynine against CYP2C9, CYP2D6, and CYP3A activity with and without NADPH, post-30-min preincubation

Values represent means \pm S.E. of the estimates obtained via nonlinear least-squares regression.

\begin{tabular}{llcc}
\hline \multirow{2}{*}{ Enzyme } & \multicolumn{2}{c}{$\mathrm{IC}_{50}(\mu \mathrm{M})$} & \\
\cline { 2 - 3 } & \multicolumn{1}{c}{$(-)-\mathrm{NADPH}$} & $(+)-\mathrm{NADPH}$ & Fold Shift \\
\hline CYP2C9 & $39.7 \pm 4.7$ & $40.0 \pm 3.2$ & 1.0 \\
CYP2D6 & $0.67 \pm 0.05$ & $0.76 \pm 0.11$ & 0.9 \\
CYP3A (HLMs) & $18.9 \pm 1.8$ & $2.6 \pm 0.3$ & 7.3 \\
CYP3A (HIMs) & $21.9 \pm 2.7$ & $3.2 \pm 0.3$ & 6.8 \\
\hline
\end{tabular}

with HLMs, the presence of NADPH during preincubation caused no leftward shift in $\mathrm{IC}_{50}$ toward CYP2C9 and CYP2D6 activities (Table 2). In contrast, the presence of NADPH during preincubation of mitragynine with HLMs or HIMs caused an $\sim 7$-fold leftward shift in $\mathrm{IC}_{50}$ toward CYP3A activity (Table 2).

Mitragynine Is a Potent Reversible Inhibitor of CYP2D6 Activity. The $\mathrm{IC}_{50}$ shift experiment showed mitragynine was a strong reversible inhibitor of CYP2D6 activity. A Lineweaver-Burk plot (reciprocal of the velocities vs. reciprocal of the substrate concentrations) indicated a competitive mode of inhibition (Fig. 2; Supplemental Data). A Dixon plot (reciprocal of velocities vs. inhibitor concentrations) was constructed to obtain an initial estimate of $K_{i}(\sim 1.2 \mu \mathrm{M})$ for nonlinear least-squares regression analysis. A competitive inhibition model best described the data, with a $K_{\mathrm{i}}$ of $1.17 \pm$ $0.15 \mu \mathrm{M}$ (Fig. 3).

Mitragynine Is a Time-Dependent Inhibitor of Hepatic and Intestinal CYP3A Activity. Both the nondilution and dilution approach confirmed mitragynine to be a time-dependent inhibitor of CYP3A activity in HLMs and HIMs. Using data from the nondilution approach, a KitzWilson plot (reciprocal of $k_{o b s}$ vs. the reciprocal of the inhibitor concentration) was constructed to obtain an initial estimate of $K_{I}(5 \mu \mathrm{M})$ and $k_{\text {inact }}\left(0.06 \mathrm{~min}^{-1}\right)$. Nonlinear least-squares regression analysis of the $k_{o b s}$ versus inhibitor concentration data produced a $K_{I}$ and $k_{\text {inact }}$ for HLMs of $4.1 \mu \mathrm{M}$ and $0.068 \mathrm{~min}^{-1}$, respectively; corresponding parameters for
HIMs were $4.2 \mu \mathrm{M}$ and $0.079 \mathrm{~min}^{-1}$, respectively. Corresponding parameters for HLMs using the dilution approach were 13.5 $\mu \mathrm{M}$ and $0.064 \mathrm{~min}^{-1}$, respectively (Fig. 4).

A Mechanistic Static Model Predicted Mitragynine to Precipitate a Clinical Pharmacokinetic Interaction with Midazolam and Other CYP3A Substrates but Not Dextromethorphan. The AUCR for dextromethorphan and midazolam when coconsumed with kratom for predominantly stimulant effects/lower dose range (1-5 g), intermediate dose range (5-8 g), and predominantly opioid-like effects/higher dose range ( $>8 \mathrm{~g}$ ) (Kruegel and Grundmann, 2018) was predicted using the average content of mitragynine contained in products $\mathrm{K}-50, \mathrm{~K}-51$, and $\mathrm{K}-52(10.32 \mathrm{mg} / \mathrm{g})$ (Todd et al., 2020 ). With a single dose of 2,5 , and $8 \mathrm{~g}$ of kratom, the AUCR for dextromethorphan using the $K_{i, u}$ value from incubations with HLMs was $1.06,1.14$, and 1.23 , respectively (Fig. 6). The predicted AUCR for midazolam using $K_{I, u}$ and $k_{\text {inact }}$ values from the nondilution incubations with HLM and HIM incubations were 5.69, 9.50, and 12.08, respectively. Based on the Food and Drug Administration-recommended cutoff (1.25) (Food and Drug Administration Center for Drug Evaluation and Research, 2020), kratom was predicted to precipitate a clinical interaction with midazolam but not dextromethorphan. Based on results with midazolam and the single 2-g dose of kratom, additional CYP3A substrates were tested that encompassed a wide range of $F_{g}$ and $f_{m}$ values. Predicted AUCRs ranged from $\sim 1.6$ to 14 (Table 3 ).

\section{Discussion}

Widespread kratom use for self-treating opioid withdrawal symptoms and pain has raised concerns among United States federal regulators, including the Drug Enforcement Administration and the Food and Drug Administration, regarding the safety of kratom, especially when combined with other drugs (https://www.fda.gov/news-events/press-announcements/ statement-fda-commissioner-scott-gottlieb-md-agencys-scientificevidence-presence-opioid-compounds). The deaths associated with kratom have been hypothesized to be due to potential kratom-drug interactions (Hughes, 2019; Veltri and Grundmann,

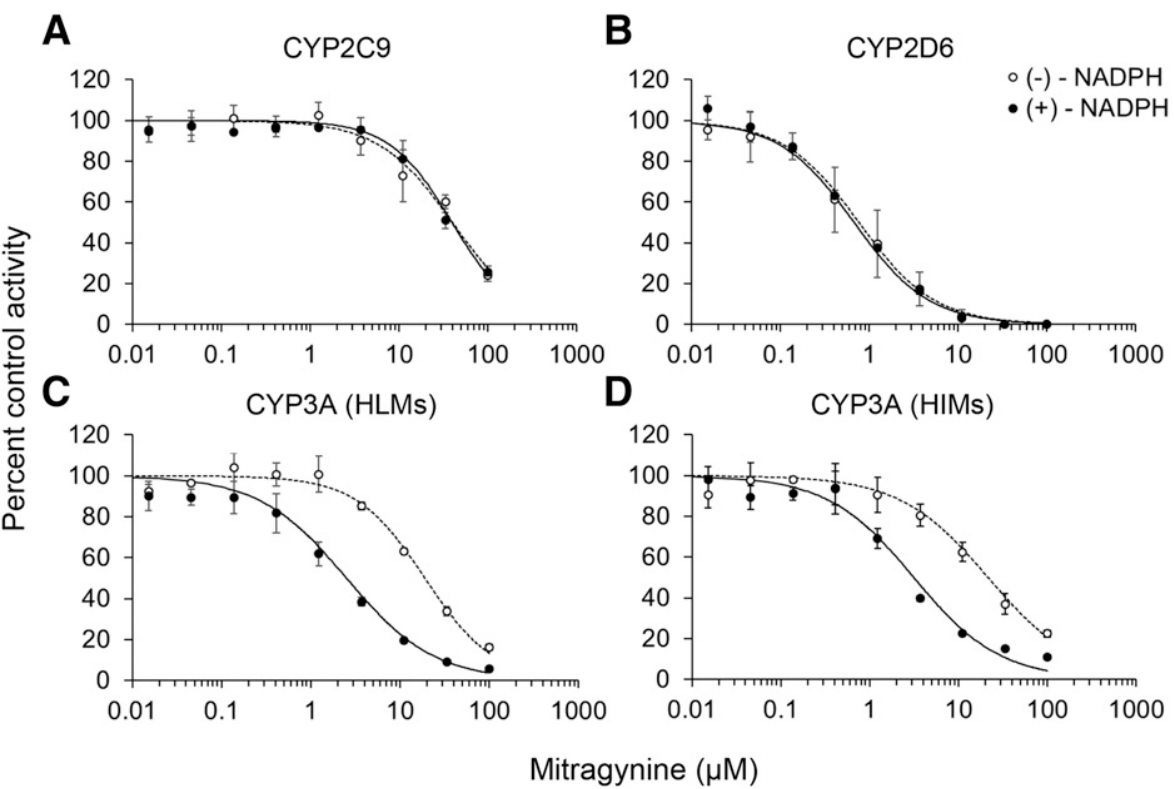

Fig. 2. $\mathrm{IC}_{50}$ curves for mitragynine after preincubation with HLMs using the index reactions diclofenac 4'-hydroxylation (CYP2C9) (A), dextromethorphan $O$-demethylation (CYP2D6) (B), and midazolam 1'-hydroxylation (CYP3A) (C) and with HIMs using midazolam 1'-hydroxylation (D) in the presence and absence of NADPH. Mitragynine was tested from 0.015 to $100 \mu$ M. Symbols and error bars denote means and S.D., respectively, of triplicate incubations. Curves denote nonlinear least-squares regression of the data. 
A

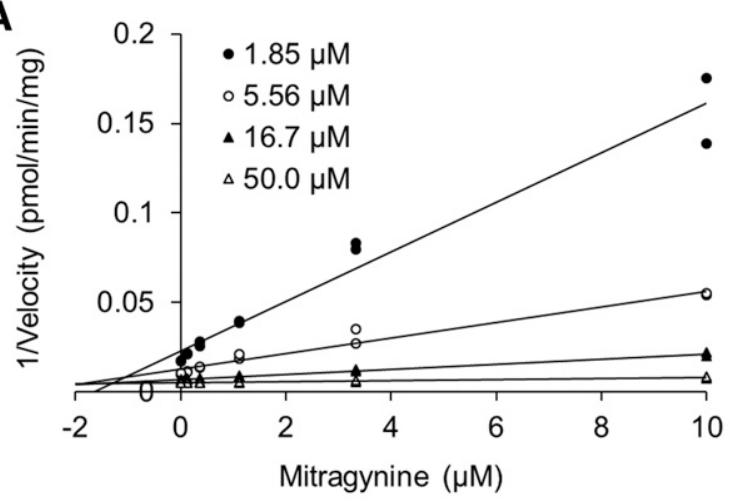

B

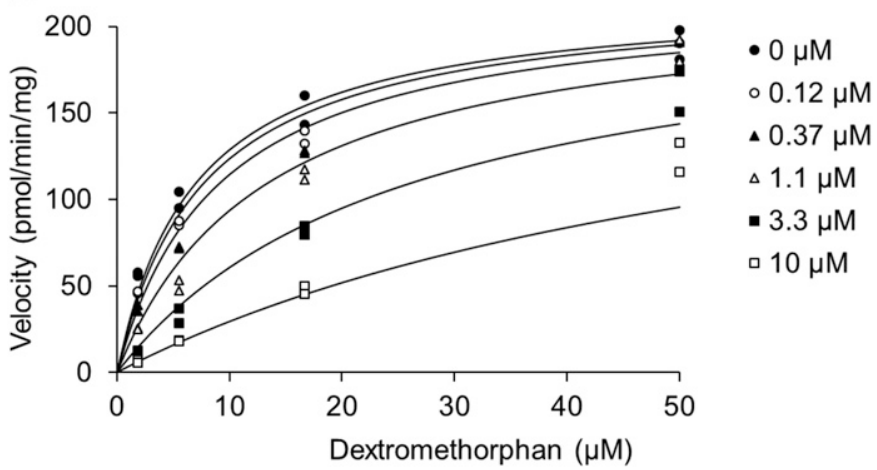

Fig. 3. Concentration-dependent inhibition of CYP2D6-mediated dextromethorphan $O$-demethylation by mitragynine $(0.37-10 \mu \mathrm{M})$ in HLMs. (A) Dixon and (B) Michaelis-Menten plots of the data. Symbols denote individual data points of duplicate incubations. Curves denote nonlinear least-squares regression of the data using the competitive inhibition model.

2019). Kratom extracts and several isolated alkaloids have been reported to inhibit CYP activity in vitro, but these observations were deemed clinically irrelevant unless very high kratom doses are consumed with CYP2D6 substrates (Hanapi et al., 2010; Kong et al., 2011; Kamble et al., 2020). However, none of these studies comprehensively investigated additional mechanisms of CYP inhibition or considered the intestine, which serves as the first site for a potential drug interaction for orally consumed xenobiotics. The objective of this work was to reassess the inhibitory potential of kratom and the marker constituent mitragynine toward hepatic CYP2C9, CYP2D6, and CYP3A and intestinal CYP3A using a systematic tiered approach that considered both reversible and time-dependent inhibition (Houston and Galetin, 2010; Pearson and Wienkers, 2019). Results enabled a more robust quantitative prediction of potential pharmacokinetic kratom-drug interactions.

Consistent with previous reports (Hanapi et al., 2010; Kong et al., 2011), all three methanolic kratom extracts strongly inhibited CYP2D6 and modestly inhibited CYP3A and CYP2C9 in HLMs in a concentration-dependent manner (Fig. 1; Supplemental Data). Likewise, all extracts showed concentration-dependent inhibition of CYP3A in HIMs, with relatively stronger effects compared to HLMs (24\%-87\% vs. $18 \%-66 \%$ inhibition). The extents of inhibition toward all CYPs were similar among the three extracts, indicating minimal interproduct variability with respect to CYP inhibition-mediated interaction potential. Mitragynine exhibited similar trends with HLMs, showing the most potent inhibition against CYP2D6, followed by CYP3A and CYP2C9. The effects of mitragynine on CYP3A were similar between HLMs and HIMs. Because mitragynine inhibited the three enzymes by $>50 \%$ at the highest concentration tested, mitragynine was advanced to the next tier of testing as a potential time-dependent inhibitor of each CYP (Fig. 1).

Consistent with previous screening data (Kamble et al., 2020), mitragynine was a modest inhibitor of CYP2C9 (IC 50 $\sim 40 \mu \mathrm{M})$. This observation, combined with the lack of a leftward shift in the $\mathrm{IC}_{50}$ upon preincubation of mitragynine with HLMs in the presence and absence of NADPH (Fig. 2), indicated mitragynine to be a modest, reversible inhibitor of CYP2C9 with minimal interaction risk. As with CYP2C9, mitragynine caused no leftward shift in $\mathrm{IC}_{50}$ against CYP2D6 (Fig. 2). However, the $\mathrm{IC}_{50}(\sim 1 \mu \mathrm{M})$ suggested mitragynine could be a clinically relevant reversible CYP2D6 inhibitor. As such, mitragynine was advanced to the next tier of testing, which was to determine the kinetics and mode of inhibition (Fig. 1). Confirming a previous report (Kamble et al., 2020), mitragynine was a strong competitive inhibitor of CYP2D6 ( $K_{i}$ $\sim 1 \mu \mathrm{M}$, Fig. 4). Using the average content of mitragynine in a 1-g dose of kratom (10.32 mg) (Todd et al., 2020) and assuming a linear relationship between the dose and $C_{\max }$ of mitragynine, the mechanistic static model predicted an interaction risk (AUCR > 1.25) with CYP2D6 substrates only at kratom doses exceeding $\sim 9 \mathrm{~g}$ (Fig. 6). The high extent of binding of mitragynine to plasma proteins $\left(f_{u, p}, 0.02\right)$ (Obeng et al., 2020) may have contributed to the low interaction risk.

Mitragynine upon preincubation with HLMs or HIMs in the presence and absence of NADPH caused an approximately 7fold leftward shift in $\mathrm{IC}_{50}$ against CYP3A activity $(\sim 20$ to $3 \mu \mathrm{M}$, Fig. 2). Because the fold shift exceeded 1.5, mitragynine was advanced to the next tier of testing to evaluate as a timedependent inhibitor. Using the nondilution approach, the inactivation efficiency $\left(k_{\text {inact }} / K_{I}\right)$ of mitragynine with both HLMs and HIMs $(\sim 17 \mathrm{ml} / \mathrm{min} / \mu \mathrm{mol})$ ranged between $k_{\text {inact }} /$ $K_{I}$ for the clinically relevant time-dependent inhibitors diltiazem $(2.7 \mathrm{ml} / \mathrm{min} / \mu \mathrm{mol})$ and verapamil $(24 \mathrm{ml} / \mathrm{min} / \mu \mathrm{mol})$ (Obach et al., 2007). Because these observations do not necessarily indicate clinical relevance, a mechanistic static model that considered TDI in both the liver and intestine was used to predict the magnitude of potential CYP3A-mediated kratom-drug interactions. Unlike for CYP2D6, even a low dose of kratom $(2 \mathrm{~g})$ was predicted to precipitate a CYP3A-mediated interaction (AUCR, 5.7). A previous report, which did not consider mitragynine as a time-dependent inhibitor nor the intestine, deemed mitragynine a reversible competitive CYP3A inhibitor and predicted no interaction risk (Hanapi et al., 2013).

The time- and concentration-dependent inhibitory effects of mitragynine against CYP3A using the dilution approach confirmed no reversibility of the observed TDI (Grimm et al., 2009; Chan et al., 2011; Parkinson et al., 2011). The lower $k_{\text {inact }} / K_{I}(4.7 \mathrm{ml} / \mathrm{min} / \mu \mathrm{mol})$ relative to that obtained from the nondilution approach was due to an approximately 3-fold higher $K_{I}$. This discrepancy could be due to partial reversal of inhibition or decreased availability of mitragynine in the primary incubation, which contained a 10 -fold higher microsomal protein concentration, leading to increased microsomal binding and/or depletion rate of mitragynine. Regardless, the interaction risk remained with a single 2 -g kratom dose (AUCR, 3.1). 

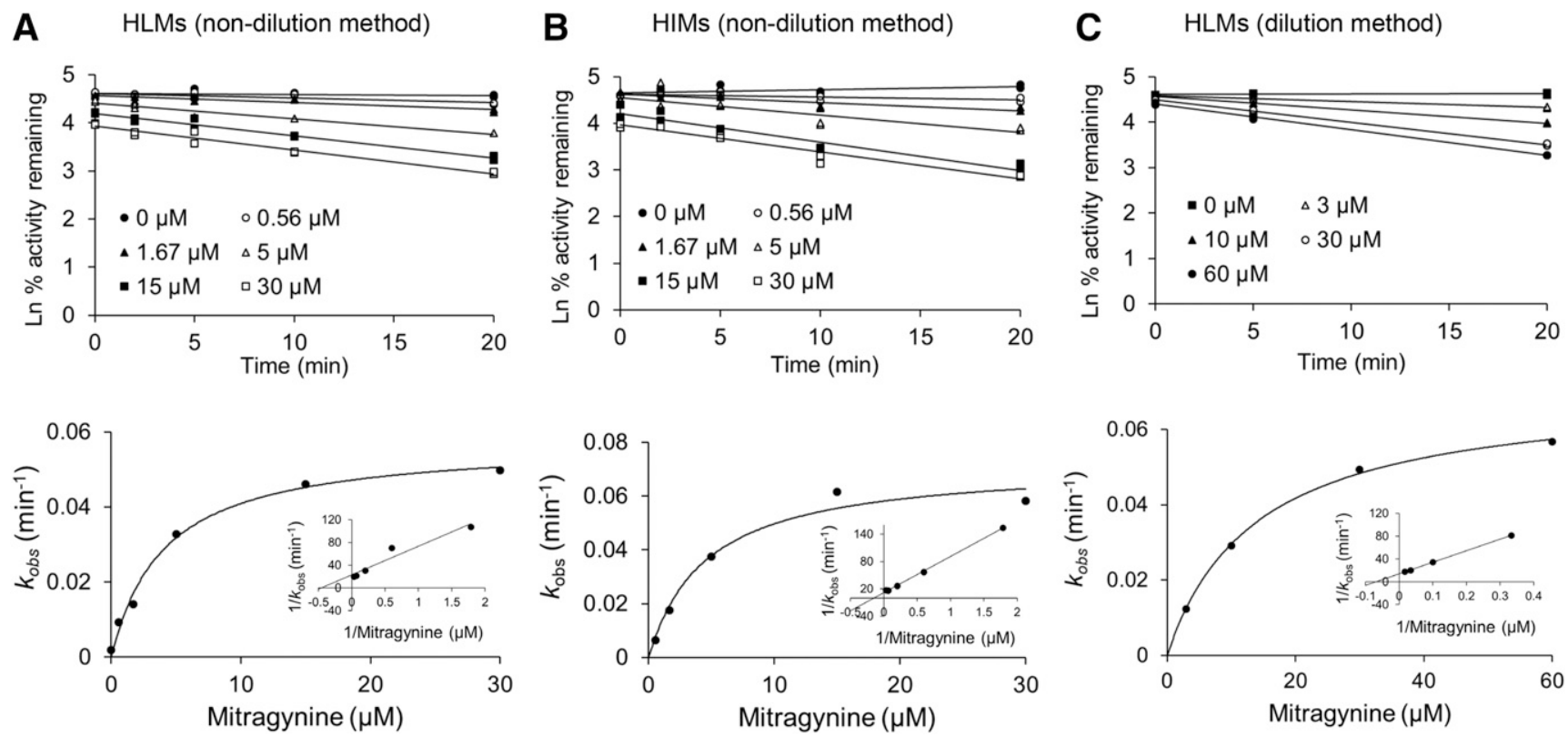

Fig. 4. Concentration- and time-dependent inhibition of CYP3A-mediated midazolam 1'-hydroxylation by mitragynine using a nondilution (1.67-30 $\mu \mathrm{M})$ method with HLMs (A) and HIMs (B) and a dilution method (3-60 $\mu \mathrm{M})$ with HLMs (C). Upper panels show log-linear decline in CYP3A activity with time. Symbols denote individual data points of duplicate incubations. Lines denote linear regression of the initial monoexponential decline. Lower panels show replots of the rate constants against inhibitor concentration. Curves denote nonlinear least-squares regression of the data. Insets depict the Kitz-Wilson plot of the time-dependent inhibition observed for mitragynine.

We speculate that mitragynine undergoes bioactivation in the microsomal incubations to form reactive species causing TDI (Kalgutkar et al., 2005) (Fig. 5). The quinolizidine moiety on mitragynine is known to undergo oxidative dehydrogenation (Kamble et al., 2019; Basiliere and Kerrigan, 2020)), which could form an imine intermediate and inactivate CYP3A by covalently binding to (a) nucleophilic residue(s) on the enzyme (Li et al., 2014). Dehydrogenation of mitragynine could also generate a highly electrophilic, 3-methylindolenine-like species as proposed for several 3-alkylindole-containing compounds, including 3-methylindole, zafirlukast, and SPD-304 (Sun and Yost, 2008; Li et al., 2014). Bioactivation of the indoloquinazoline alkaloids evodiamine and rutaecarpine to inactivate CYP3A4 was proposed to occur via the 3-methylindolenine intermediate (Wen et al., 2014). Another mechanism could involve bioactivation of mitragynine through formation of a $p$-quinone intermediate via sequential oxidative $O$-demethylation, aromatic hydroxylation, and dehydrogenation of the indoloquinolizidine moiety; however, this mechanism requires three discrete oxidation steps. Alternatively, a two-step mechanism involving aromatic hydroxylation and oxidative dehydrogenation could include formation of a reactive $o$-quinoneimine (Fig. 5). Nucleophilic residues on the apoprotein can form covalent adducts with such intermediates, inactivating the enzyme. Additional biochemical experiments are needed to ascertain the exact mechanism of CYP3A TDI by mitragynine.

Regardless of the mechanism, TDI of CYP3A in the liver and/or intestine could explain the attainment of toxic concentrations of the CYP3A substrate quetiapine described in a case report of a kratom-related death in which quetiapine overdose was not suspected (Hughes, 2019). The AUCR for quetiapine, which undergoes extensive first-pass metabolism in the liver, was predicted to be $\sim 2.5$-fold when taken with a 2-g dose of kratom (Table 3), supporting the hypothesis that the kratomrelated death could be due to TDI of CYP3A. Based on results with midazolam and quetiapine, the interaction risk for other

TABLE 3

Prediction of CYP3A-mediated kratom-drug interactions via a mechanistic static model

The object drugs were selected to encompass a wide range of $F_{g}$ and $f_{m}$ values with minimal influence of transporters.

\begin{tabular}{lcccr}
\hline Victim Drug & Therapeutic Drug Class & $F_{g}$ (Galetin et al., 2008) & $f_{m}$ (Shou et al., 2008) & AUCR \\
\hline Alfentanil & Opioid, analgesic & 0.6 & 0.87 & 4.26 \\
Alprazolam & Anxiolytic & 0.94 & 0.8 & 2.43 \\
Buspirone & Anxiolytic & 0.21 & 0.95 & 14.04 \\
Cyclosporine & Immunosuppressant & 0.44 & 0.71 & 4.5 \\
Felodipine & Antianginal & 0.45 & $0.81^{a}$ & 5.12 \\
Nifedipine & Antianginal & 0.78 & 0.84 & 2.66 \\
Quetiapine & Antipsychotic & $0.99^{b}$ & 0.76 & 2.46 \\
Quinidine & Antiarrhythmic & 0.9 & $0.35^{c}$ & 2.38 \\
Trazodone & Antidepressant & 0.83 & 0.92 & 1.60 \\
Triazolam & Hypnotic & 0.75 & 0.26 & 3.76 \\
Zolpidem & Hypnotic & 0.79 & & 1.55 \\
\hline
\end{tabular}

${ }^{a}$ Yadav et al. (2018)

${ }^{b}$ Mano et al. (2015).

${ }^{c}$ Mao et al. (2011). 

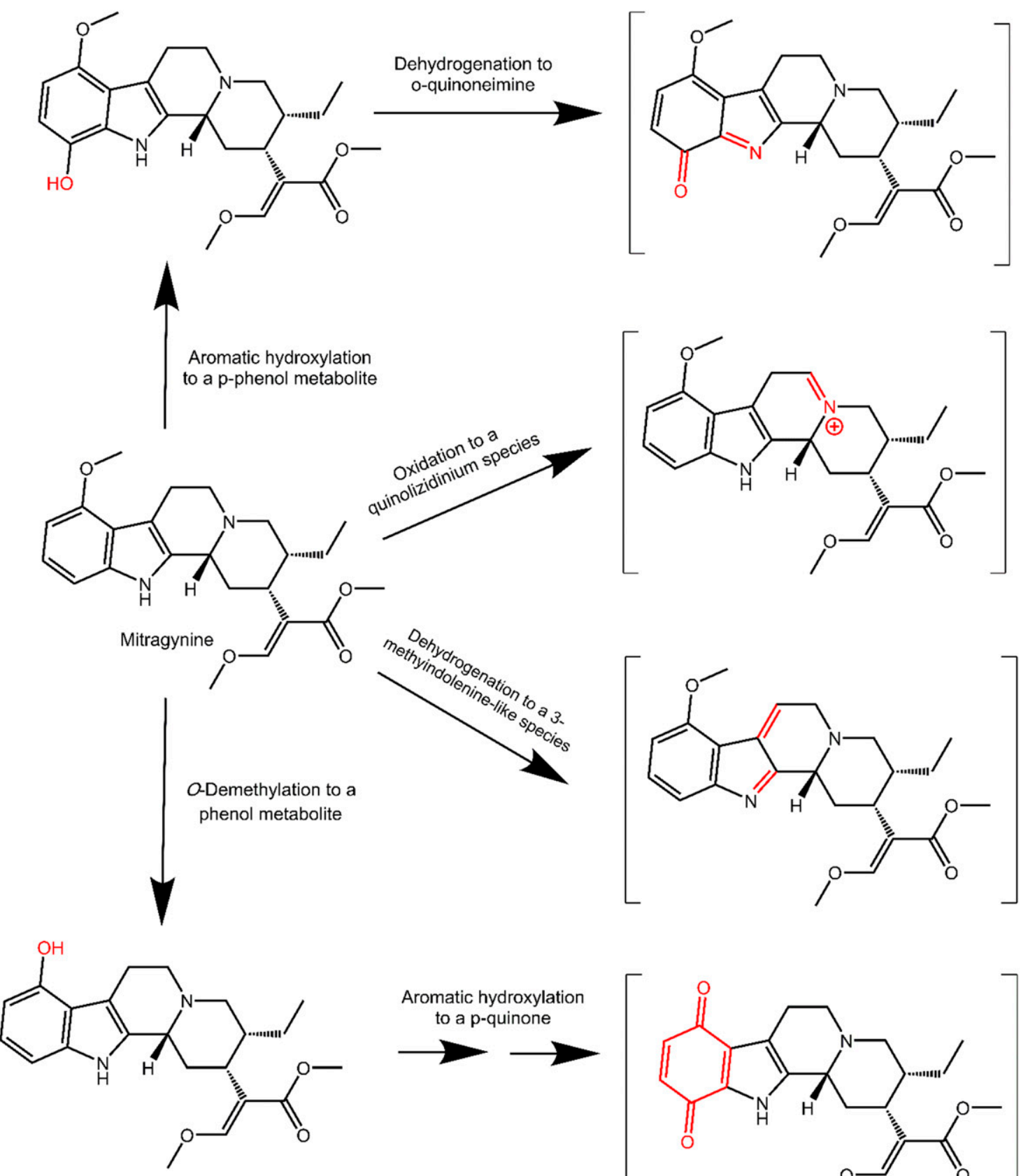

Fig. 5. Speculated bioactivation mechanisms for mitragynine causing time-dependent inhibition of CYP3A activity (midazolam 1'-hydroxylation).

CYP3A substrates was predicted using the same dose of kratom. All AUCR predictions ranged from 1.6 (zolpidem) to 14 (buspirone), warranting further evaluation to de-risk these potential pharmacokinetic interactions.

The interaction risk for kratom is expected to be higher at higher doses, particularly given that $K_{i}$ and $K_{I}$ of mitragynine toward CYP2D6 and CYP3A were within concentrations reported for autopsy blood samples from kratom-related deaths (1-13 vs. 0.04-12 $\mu \mathrm{M})$. Although mitragynine has been reported to bind extensively to plasma proteins $\left(f_{u, p}\right.$, 0.02-0.15) (Kong et al., 2017; Obeng et al., 2020), the partitioning of mitragynine from blood into the liver, as well as whether uptake transporters influence intracellular mitragynine concentrations, is not known. The majority of these decedents (14 of 15) tested positive for opioids and/or benzodiazepines, several of which are metabolized by CYP2D6 and CYP3A (Table 1), further supporting kratom as a potential precipitant of adverse pharmacokinetic drug interactions. Notably, risk of kratom-opioid interactions could be on a rise parallel to the "third wave" of the opioid epidemic dominated by fentanyl and other illicitly manufactured fentanyl analogs (https://www.cdc.gov/drugoverdose/epidemic/index.html). In fact, fentanyl and its analogs, which are mainly metabolized by CYP3A, were listed as causing unintentional overdose deaths in approximately 65\% (99 of 152) of kratom-positive decedents (Olsen et al., 2019). 


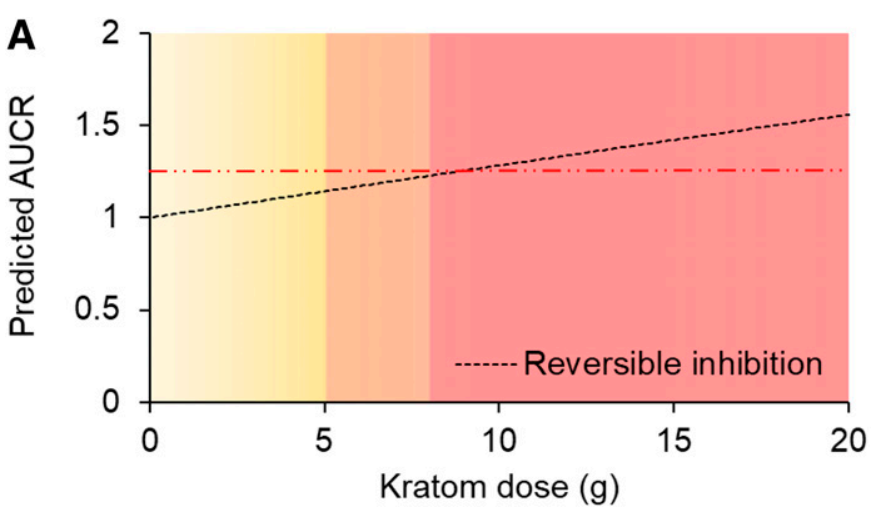

B

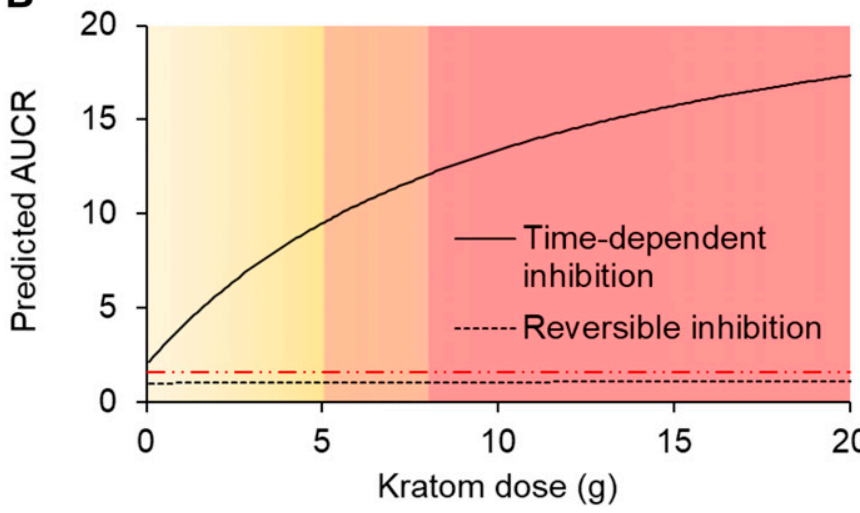

Fig. 6. Drug interaction risks predicted via mechanistic static models associated with kratom use for stimulant effects/lower dose range (1-5 g), intermediate dose range (5-8 g), and predominantly opioid-like effects/ higher dose range $(>8 \mathrm{~g}$ ). An interaction risk (AUCR $>1.25$, dashed red line) via reversible inhibition of CYP2D6 (dextromethorphan $O$-demethylation activity) (A) and reversible (from previous reports, dashed black line) and time-dependent (from current work, solid black line) inhibition of CYP3A (midazolam 1'-hydroxylation activity) (B).

There are limitations to the current work. First, only mitragynine was tested as a candidate CYP inhibitor. The concentration of mitragynine in the kratom extracts at $20 \mu \mathrm{g} / \mathrm{ml}$ was approximately $1.2 \mu \mathrm{M}$. Compared with purified mitragynine at $1 \mu \mathrm{M}$, the extracts showed stronger inhibition against the three enzymes tested (Fig. 1; Supplemental Data). Other alkaloids, including paynantheine and speciogynine, which combined represent $\sim 15 \%$ of total alkaloid content, may contribute to potential kratom-drug interactions. Second, the mechanism of CYP3A TDI by mitragynine remains unknown and is needed to confidently estimate the severity of a potential interaction. Studies are underway to identify the mechanism(s). Third, secondary glucuronide and sulfate conjugates of mitragynine have been reported in human urine upon kratom consumption (Philipp et al., 2009). Such phase II metabolism could compete with the formation of reactive intermediates responsible for CYP3A TDI, reducing interaction risk. Whole cell systems, including hepatocytes and enterocytes, could be used to test this hypothesis. Fourth, mechanistic static models, which are useful for early decision-making, such as in the current work, were used to predict interaction risk. Once robust human mitragynine pharmacokinetic data are obtained, a dynamic (i.e., physiologically-based pharmacokinetic) interaction model will be developed to predict the time course, gain additional mechanistic insight, and extrapolate to other CYP2D6- and CYP3A-sensitive object drugs.
In summary, using a systematic tiered approach that considered both reversible and TDI and both the liver and intestine as sites of potential drug interactions, mitragynine was identified as a potent reversible inhibitor of CYP2D6 and a time-dependent inhibitor of CYP3A. Extrapolation of the current in vitro to in vivo predictions to real-world scenarios involving other drugs, as well as varied mitragynine content among different kratom products and kratom consumption patterns, can be realized through physiologically-based pharmacokinetic modeling and simulation and clinical assessment. Such definitive information will help address ongoing concerns from regulatory authorities regarding the safety of kratom when coconsumed with drugs.

\section{Acknowledgments}

M.F.P. dedicates this article to Dr. David P. Paine.

\section{Authorship Contributions}

Participated in research design: Tanna, Tian, Rettie, Thummel, Paine.

Conducted experiments: Tanna.

Contributed new reagents or analytic tools: Cech, Oberlies.

Performed data analysis: Tanna, Tian, Paine.

Wrote or contributed to the writing of the manuscript: Tanna, Tian, Cech, Oberlies, Rettie, Thummel, Paine.

\section{References}

Ainslie GR, Wolf KK, Li Y, Connolly EA, Scarlett YV, Hull JH, and Paine MF (2014) Assessment of a candidate marker constituent predictive of a dietary substancedrug interaction: case study with grapefruit juice and CYP3A4 drug substrates. $J$ Pharmacol Exp Ther 351:576-584.

Basiliere S and Kerrigan S (2020) Temperature and pH-dependent stability of mitragyna alkaloids. J Anal Toxicol 44:314-324.

Chan CY, New LS, Ho HK, and Chan ECY (2011) Reversible time-dependent inhibition of cytochrome P450 enzymes by duloxetine and inertness of its thiophene ring towards bioactivation. Toxicol Lett 206:314-324.

Chang-Chien GC, Odonkor CA, and Amorapanth P (2017) Is Kratom the new 'legal high'on the block?: the case of an emerging opioid receptor agonist with substance abuse potential. Pain Physician 20:E195-E198.

Cinosi E, Martinotti G, Simonato P, Singh D, Demetrovics Z, Roman-Urrestarazu A, Bersani FS, Vicknasingam B, Piazzon G, Li J-H, et al. (2015) Following "the roots" of Kratom (Mitragyna speciosa): the evolution of an enhancer from a traditional use to increase work and productivity in Southeast Asia to a recreational psychoactive drug in western countries. BioMed Res Int 2015:968786.

Crews KR, Gaedigk A, Dunnenberger HM, Leeder JS, Klein TE, Caudle KE, Haidar CE, Shen DD, Callaghan JT, Sadhasivam S, et al.; Clinical Pharmacogenetics Implementation Consortium (2014) Clinical Pharmacogenetics Implementation Consortium guidelines for cytochrome P450 2D6 genotype and codeine therapy: 2014 update. Clin Pharmacol Ther 95:376-382.

Ewald AH and Maurer HH (2008) 2,5-Dimethoxyamphetamine-derived designer drugs: studies on the identification of cytochrome P450 (CYP) isoenzymes involved in formation of their main metabolites and on their capability to inhibit CYP2D6. Toxicol Lett 183:52-57.

Fahmi OA, Hurst S, Plowchalk D, Cook J, Guo F, Youdim K, Dickins M, Phipps A, Darekar A, Hyland R, et al. (2009) Comparison of different algorithms for predicting clinical drug-drug interactions, based on the use of CYP3A4 in vitro data: predictions of compounds as precipitants of interaction. Drug Metab Dispos 37:1658-1666.

Food and Drug Administration Centre for Drug Evaluation and Research (2020), In Vitro Drug Interaction Studies - Cytochrome P450 Enzyme- and TransporterMediated Drug Interactions Guidance for Industry, U.S. Food and Drug Administration, Silver Spring, MD.

Flores-Bocanegra L, Raja HA, Graf TN, Augustinović M, Wallace ED, Hematian S, Kellogg JJ, Todd DA, Cech NB, and Oberlies NH (2020) The chemistry of kratom [Mitragyna speciosa]: updated characterization data and methods to elucidate indole and oxindole alkaloids. $J$ Nat Prod 83:2165-2177.

Galetin A, Gertz M, and Houston JB (2008) Potential role of intestinal first-pass metabolism in the prediction of drug-drug interactions. Expert Opin Drug Metab Toxicol 4:909-922.

Gershman K, Timm K, Frank M, Lampi L, Melamed J, Gerona R, and Monte AA (2019) Deaths in Colorado attributed to kratom. N Engl J Med 380:97-98.

Gjestad C, Haslemo T, Andreassen OA, and Molden E (2017) 4 $\beta$-Hydroxycholesterol level significantly correlates with steady-state serum concentration of the CYP3A4 substrate quetiapine in psychiatric patients. Br J Clin Pharmacol 83:2398-2405.

Grimm SW, Einolf HJ, Hall SD, He K, Lim H-K, Ling K-HJ, Lu C, Nomeir AA, Seibert $\mathrm{E}$, Skordos KW, et al. (2009) The conduct of in vitro studies to address timedependent inhibition of drug-metabolizing enzymes: a perspective of the pharmaceutical research and manufacturers of America. Drug Metab Dispos 37:1355-1370.

Gufford BT, Chen G, Lazarus P, Graf TN, Oberlies NH, and Paine MF (2014) Identification of diet-derived constituents as potent inhibitors of intestinal glucuronidation. Drug Metab Dispos 42:1675-1683. 
Gufford BT, Chen G, Vergara AG, Lazarus P, Oberlies NH, and Paine MF (2015) Milk thistle constituents inhibit raloxifene intestinal glucuronidation: a potential clinically relevant natural product-drug interaction. Drug Metab Dispos 43:1353-1359.

Guitton J, Buronfosse T, Désage M, Lepape A, Brazier J-L, and Beaune P (1997) Possible involvement of multiple cytochrome P450S in fentanyl and sufentanil metabolism as opposed to alfentanil. Biochem Pharmacol 53:1613-1619.

Hanapi N, Azizi J, Ismail S, and Mansor S (2010) Evaluation of selected Malaysian medicinal plants on phase I drug metabolizing enzymes, CYP 2C9, CYP2D6 and CYP3A4 activities in vitro. Int J Pharmacol 6:494-499.

Hanapi NA, Ismail S, and Mansor SM (2013) Inhibitory effect of mitragynine on human cytochrome P450 enzyme activities. Pharmacognosy Res 5:241-246.

Hassan Z, Muzaimi M, Navaratnam V, Yusoff NH, Suhaimi FW, Vadivelu R, Vicknasingam BK, Amato D, von Hörsten S, Ismail NI, et al. (2013) From Kratom to mitragynine and its derivatives: physiological and behavioural effects related to use, abuse, and addiction. Neurosci Biobehav Rev 37:138-151.

Henningfield JE, Fant RV, and Wang DW (2018) The abuse potential of kratom according the 8 factors of the controlled substances act: implications for regulation and research. Psychopharmacology (Berl) 235:573-589.

Houston JB and Galetin A (2010) In vitro techniques to study drug-drug interactions of drug metabolism: cytochrome P450, in Enzyme-and Transporter-Based Drug-Drug Interactions: Progress and Future Challenges (Pang KS, Rodrigues AD, and Peter RM eds), pp 169-215, Springer, NY.

Hughes RL (2019) Fatal combination of mitragynine and quetiapine - a case report with discussion of a potential herb-drug interaction. Forensic Sci Med Pathol 15:110-113.

Johnson E.J, González-Peréz V, Tian D-D, Lin YS, Unadkat JD, Rettie AE, Shen DD McCune JS, and Paine MF (2018) Selection of priority natural products for evaluation as potential precipitants of natural product-drug interactions: a NaPDI center recommended approach. Drug Metab Dispos 46:1046-1052.

Kalgutkar AS, Gardner I, Obach RS, Shaffer CL, Callegari E, Henne KR, Mutlib AE, Dalvie DK, Lee JS, Nakai Y, et al. (2005) A comprehensive listing of bioactivation pathways of organic functional groups. Curr Drug Metab 6:161-225.

Kamble SH, Sharma A, King TI, Berthold EC, León F, Meyer PKL, Kanumuri SRR McMahon LR, McCurdy CR, and Avery BA (2020) Exploration of cytochrome P450 inhibition mediated drug-drug interaction potential of kratom alkaloids. Toxicol Lett 319:148-154.

Kamble SH, Sharma A, King TI, León F, McCurdy CR, and Avery BA (2019) Metabolite profiling and identification of enzymes responsible for the metabolism of mitragynine, the major alkaloid of Mitragyna speciosa (kratom). Xenobiotica 49:1279-1288.

Kamendulis LM, Brzezinski MR, Pindel EV, Bosron WF, and Dean RA (1996) Metabolism of cocaine and heroin is catalyzed by the same human liver carboxylesterases. J Pharmacol Exp Ther 279:713-717.

Kanamori T, Iwata YT, Segawa H, Yamamuro T, Kuwayama K, Tsujikawa K, and Inoue $\mathrm{H}$ (2019) Metabolism of butyrylfentanyl in fresh human hepatocytes: chemical synthesis of authentic metabolite standards for definitive identification. Biol Pharm Bull 42:623-630.

Khalil S, Abdullah SAJ, and Ahmad R (2020) Enforcement status of the poison act 1952 against offences related to kratom (Mitragyna speciosa korth) misuse in Malaysia. UUM J Legal Studies 11:75-93.

Kobayashi K, Ishizuka T, Shimada N, Yoshimura Y, Kamijima K, and Chiba K (1999) Sertraline N-demethylation is catalyzed by multiple isoforms of human cytochrome P-450 in vitro. Drug Metab Dispos 27:763-766.

Kong WM, Chik Z, Mohamed Z, and Alshawsh MA (2017) Physicochemical characterization of Mitragyna speciosa alkaloid extract and Mitragynine using in vitro high throughput assays. Comb Chem High Throughput Screen 20:796-803.

Kong WM, Chik Z, Ramachandra M, Subramaniam U, Aziddin RER, and Mohamed Z (2011) Evaluation of the effects of Mitragyna speciosa alkaloid extract on cytochrome P450 enzymes using a high throughput assay. Molecules 16:7344-7356.

Kruegel AC, Gassaway MM, Kapoor A, Váradi A, Majumdar S, Filizola M, Javitch JA, and Sames D (2016) Synthetic and receptor signaling explorations of the Mitragyna alkaloids: mitragynine as an atypical molecular framework for opioid receptor modulators. J Am Chem Soc 138:6754-6764.

Kruegel AC and Grundmann O (2018) The medicinal chemistry and neuropharmacology of kratom: a preliminary discussion of a promising medicinal plant and analysis of its potential for abuse. Neuropharmacology 134:108-120.

Lalovic B, Phillips B, Risler LL, Howald W, and Shen DD (2004) Quantitative contribution of CYP2D6 and CYP3A to oxycodone metabolism in human liver and intestinal microsomes. Drug Metab Dispos 32:447-454.

Li AC, Yu E, Ring SC, and Chovan JP (2014) Structural identification of imatinib cyanide adducts by mass spectrometry and elucidation of bioactivation pathway. Rapid Commun Mass Spectrom 28:123-134.

LLerena A, Dorado P, Berecz R, González AP, and Peñas-LLedó EM (2004) Effect of CYP2D6 and CYP2C9 genotypes on fluoxetine and norfluoxetine plasma concentrations during steady-state conditions. Eur J Clin Pharmacol 59:869-873.

Mano Y, Sugiyama Y, and Ito K (2015) Use of a physiologically based pharmacokinetic model for quantitative prediction of drug-drug interactions via CYP3A4 and estimation of the intestinal availability of CYP3A4 substrates. J Pharm Sci 104:3183-3193.

Mao J, Mohutsky MA, Harrelson JP, Wrighton SA, and Hall SD (2011) Prediction of CYP3A-mediated drug-drug interactions using human hepatocytes suspended in human plasma. Drug Metab Dispos 39:591-602.

McDonald MG, Au NT, and Rettie AE (2015) P450-based drug-drug interactions of amiodarone and its metabolites: diversity of inhibitory mechanisms. Drug Metab Dispos 43:1661-1669.

McDonald MG, Tian D-D, Thummel KE, Paine MF, and Rettie AE (2020) Modulation of major human liver microsomal cytochromes P450 by component alkaloids of goldenseal: time-dependent inhibition and allosteric effects. Drug Metab Dispos 48 1018-1027.

Miotto K, Cho AK, Khalil MA, Blanco K, Sasaki JD, and Rawson R (2017) Trends in tramadol: pharmacology, metabolism, and misuse. Anesth Analg 124:44-51.

Niwa T, Shiraga T, Ishii I, Kagayama A, and Takagi A (2005) Contribution of human hepatic cytochrome p450 isoforms to the metabolism of psychotropic drugs. Biol Pharm Bull 28:1711-1716.
Obach RS, Walsky RL, and Venkatakrishnan K (2007) Mechanism-based inactivation of human cytochrome p450 enzymes and the prediction of drug-drug interactions. Drug Metab Dispos 35:246-255.

Obeng S, Kamble SH, Reeves ME, Restrepo LF, Patel A, Behnke M, Chear NJ-Y, Ramanathan S, Sharma A, León F, et al. (2020) Investigation of the adrenergic and opioid binding affinities, metabolic stability, plasma protein binding properties, and functional effects of selected indole-based kratom alkaloids. J Med Chem 63:433-439.

Olsen EOM, O'Donnell J, Mattson CL, Schier JG, and Wilson N (2019) Notes from the field: unintentional drug overdose deaths with kratom detected-27 states, July 2016-December 2017. MMWR Morb Mortal Wkly Rep 68:326-327.

Ono S, Hatanaka T, Miyazawa S, Tsutsui M, Aoyama T, Gonzalez FJ, and Satoh T (1996) Human liver microsomal diazepam metabolism using cDNA-expressed cytochrome P450s: role of CYP2B6, 2C19 and the 3A subfamily. Xenobiotica 26:1155-1166.

Paine MF, Shen DD, and McCune JS (2018) Recommended approaches for pharmacokinetic natural product-drug interaction research: a NaPDI center commentary. Drug Metab Dispos 46:1041-1045.

Parkinson A, Kazmi F, Buckley DB, Yerino P, Paris BL, Holsapple J, Toren P, Otradovec SM, and Ogilvie BW (2011) An evaluation of the dilution method for identifying metabolism-dependent inhibitors of cytochrome P450 enzymes. Drug Metab Dispos 39:1370-1387.

Parmentier Y, Pothier C, Hewitt N, Vincent L, Caradec F, Liu J, Lin F, Trancart M-M, Guillet F, Bouaita B, et al. (2019) Direct and quantitative evaluation of the major human CYP contribution (fmCYP) to drug clearance using the in vitro Silensomes ${ }^{\mathrm{TM}}$ model. Xenobiotica 49:22-35.

Pearson PG and Wienkers LC (2019) Handbook of Drug Metabolism, CRC Press, FL. Philipp AA, Wissenbach DK, Zoerntlein SW, Klein ON, Kanogsunthornrat J, and Maurer HH (2009) Studies on the metabolism of mitragynine, the main alkaloid of the herbal drug Kratom, in rat and human urine using liquid chromatographylinear ion trap mass spectrometry. J Mass Spectrom 44:1249-1261.

Pichard L, Gillet G, Bonfils C, Domergue J, Thénot J-P, and Maurel P (1995) Oxidative metabolism of zolpidem by human liver cytochrome P450S. Drug Metab Dispos 23:1253-1262.

Post S, Spiller HA, Chounthirath T, and Smith GA (2019) Kratom exposures reported to United States poison control centers: 2011-2017. Clin Toxicol (Phila) 57:847-854.

Ramsden D, Fung C, Hariparsad N, Kenny JR, Mohutsky MA, Parrott N, Robertson S, and Tweedie DJ (2019) Perspectives from the IQ induction working group on factors impacting clinical DDI due to induction: focus on CYP3A substrates [published ahead of print]. Drug Metab Dispos DOI: 10.1124/dmd.119.087270.

Saravanakumar A, Sadighi A, Ryu R, and Akhlaghi F (2019) Physicochemical properties, biotransformation, and transport pathways of established and newly approved medications: a systematic review of the top 200 most prescribed drugs vs. the FDAapproved drugs between 2005 and 2016. Clin Pharmacokinet 58:1281-1294.

Shou M, Hayashi M, Pan Y, Xu Y, Morrissey K, Xu L, and Skiles GL (2008) Modeling, prediction, and in vitro in vivo correlation of CYP3A4 induction. Drug Metab Dispos 36:2355-2370.

Störmer E, von Moltke LL, Shader RI, and Greenblatt DJ (2000) Metabolism of the antidepressant mirtazapine in vitro: contribution of cytochromes P-450 1A2, 2D6, and 3A4. Drug Metab Dispos 28:1168-1175.

Sun $\mathrm{H}$ and Yost GS (2008) Metabolic activation of a novel 3-substituted indolecontaining TNF- $\alpha$ inhibitor: dehydrogenation and inactivation of CYP3A4. Chem Res Toxicol 21:374-385.

Throckmorton DC, Gottlieb S, and Woodcock J (2018) The FDA and the next wave of drug abuse-proactive pharmacovigilance. N Engl J Med 379:205-207.

Tian D-D, Kellogg JJ, Okut N, Oberlies NH, Cech NB, Shen DD, McCune JS, and Paine MF (2018) Identification of intestinal UDP-glucuronosyltransferase inhibitors in green tea (Camellia sinensis) using a biochemometric approach: application to raloxifene as a test drug via in vitro to in vivo extrapolation. Drug Metab Dispos 46:552-560.

Todd D A, Kellogg J J, Wallace E D, Khin M, Flores-Bocanegra L, Tanna R S, McIntosh S, Raja H A, Graf T N, Hemby S E, et al. (2020) Chemical composition and biological effects of kratom (Mitragyna speciosa): In vitro studies with implications for efficacy and drug interactions. Sci Rep 10 (1):19158, doi: 10.1038/ 441598-020-76119-w 33154449.

Tóth K, Csukly G, Sirok D, Belic A, Kiss Á, Háfra E, Déri M, Menus Á, Bitter I, and Monostory K (2016) Optimization of clonazepam therapy adjusted to patient's CYP3A status and NAT2 genotype. Int $J$ Neuropsychopharmacol 19:pyw083.

Urichuk L, Prior TI, Dursun S, and Baker G (2008) Metabolism of atypical antipsychotics: involvement of cytochrome p450 enzymes and relevance for drug-drug interactions. Curr Drug Metab 9:410-418.

Veltri C and Grundmann O (2019) Current perspectives on the impact of Kratom use. Subst Abuse Rehabil 10:23-31.

von Moltke LL, Greenblatt DJ, Giancarlo GM, Granda BW, Harmatz JS, and Shader RI (2001) Escitalopram (S-citalopram) and its metabolites in vitro: cytochromes mediating biotransformation, inhibitory effects, and comparison to R-citalopram. Drug Metab Dispos 29:1102-1109.

Wen B, Roongta V, Liu L, and Moore DJ (2014) Metabolic activation of the indoloquinazoline alkaloids evodiamine and rutaecarpine by human liver microsomes: dehydrogenation and inactivation of cytochrome P450 3A4. Drug Metab Dispos 42:1044-1054.

Williams RS and Nikitin D (2020) The internet market for Kratom, an opioid alternative and variably legal recreational drug. Int J Drug Policy 78:102715.

Yadav J, Korzekwa K, and Nagar S (2018) Improved predictions of drug-drug interactions mediated by time-dependent inhibition of CYP3A. Mol Pharm 15:1979-1995. Yamamoto Y, Takahashi Y, Imai K, Kagawa Y, and Inoue Y (2017) Effect of CYP inducers/inhibitors on topiramate concentration: clinical value of therapeutic drug monitoring. Ther Drug Monit 39:55-61.

Address correspondence to: Dr. Mary F. Paine, Department of Pharmaceutical Sciences, College of Pharmacy and Pharmaceutical Sciences, Washington State University, 412 E Spokane Falls Blvd., Spokane, WA 99202. E-mail: mary.paine@wsu.edu 